\title{
Génétique du discours périgraphique dibien
}

Sur une préface allographe et deux postfaces autographes

\section{Guy Dugas}

\section{(2) OpenEdition}

\section{Journals}

Édition électronique

URL : http://journals.openedition.org/coma/595

DOI : $10.4000 /$ coma.595

ISSN : 2275-1742

\section{Éditeur}

Institut des textes \& manuscrits modernes (ITEM)

Référence électronique

Guy Dugas, « Génétique du discours périgraphique dibien », Continents manuscrits [En ligne], 5 | 2015, mis en ligne le 15 octobre 2015, consulté le 19 avril 2019. URL : http://journals.openedition.org/ coma/595; DOI : 10.4000/coma.595

Ce document a été généré automatiquement le 19 avril 2019

\section{(c) (†) $\ominus$}

Continents manuscrits - Génétique des textes littéraires - Afrique, Caraîbe, dispora est mis à disposition selon les termes de la licence Creative Commons Attribution - Pas d'Utilisation Commerciale - Pas de Modification 4.0 International. 


\section{Génétique du discours périgraphique dibien}

Sur une préface allographe et deux postfaces autographes

\section{Guy Dugas}

1 En tant que comparatiste, je me suis toujours intéressé au discours péritextuel dans la mesure où d'est un élément qui relie l'œuvre au monde qui l'entoure. Mais pourquoi la génétique, qui se consacre essentiellement à la naissance et au développement de l'œuvre elle-même, devrait-elle s'y intéresser?

On se souvient de la définition que donne Genette du péritexte : «Ce par quoi un texte se fait livre. " Le travail que nous sommes en train d'achever sur les Portraits de Memmi m'a convaincu, si tant est que ma formation de comparatiste ne l'ait pas fait avant cela, qu'il est nécessaire lorsqu'on s'attache à la naissance et à la vie d'un livre de concevoir une génétique de son discours périgraphique ${ }^{1}$. C'est indéniablement le cas pour les textes de fiction comme pour les essais sociologiques de Memmi, dont tout un discours d'escorte, qu'il soit autographique ou allographique, oriente durablement la lecture et la réception. A tel point qu'un énoncé d'escorte s'est établi à jamais dans l'esprit du lecteur, au risque de quelques approximations : La Statue de sel, préface d'Albert Camus ; Portrait du colonisé, précédé d'un Portrait du colonisateur, préface de Jean-Paul Sartre, ou encore, au moment de la Révolution tranquille au Québec, cette réédition du Portrait du colonisé, illisible sans sa postface autographe : « Les Canadiens français sont-ils des colonisés? »

2 Je n'ai pas l'intention de parler ici des œuvres de Memmi mais de celles de Dib dont le discours d'escorte est beaucoup moins lourd et sans doute génétiquement moins décisif. Je ne prétends donc pas a priori qu'il soit de semblable importance pour lui. Ceci dit, nous ne devons pas non plus négliger a priori cet aspect de ses livres.

J'ai donc choisi de présenter successivement quelques éléments du péritexte autographe - plus précisément deux postfaces très différentes l'une de l'autre : à Qui se souvient de la mer (1962) et à La Nuit sauvage (1995) - ainsi qu'une préface allographe remarquable, celle donnée par Aragon au recueil Ombre gardienne (1961) - unique intellectuel, à ma connaissance, dont Dib ait accepté le parrainage. Cela afin de montrer l'importance de ce discours péritextuel dans l'élaboration comme dans la réception du discours dibien. 


\section{Deux postfaces autographes}

la mer et à La Nuit sauvage, longs développements explicatifs dans les deux cas mais fonctionnant, il me semble, selon des modalités assez différentes. Deux postfaces mais aucune préface dans l'œuvre de Dib: cela mérite bien une explication liminaire sur le statut de l'une et l'autre.

Permettant un dialogue avec le lecteur, préfaces et postfaces autographes agissent comme des variables d'ajustement de l'œuvre, dans une sorte d'interactivité avec le lecteur réel ou virtuel. Mais selon un mode de fonctionnement différent du fait de leur rapport au texte : placée avant, la préface constitue dans l'esprit de l'auteur un préalable à la lecture du texte, même si le lecteur est libre de choisir une autre stratégie et d'entrer directement dans ce texte; dans ce cas, elle «s'adresse à un lecteur imaginaire, qui a déjà lu» le livre qu'elle anticipe. C'est pourquoi Antoine Compagnon la définit comme un "genre impossible $»^{2}$.

La postface au contraire figure toujours comme un addendum, parfois tardif (à la suite d'une réimpression ou d'une réédition). Dans son étude intitulée Seuils ${ }^{3}$, résultant d'une large enquête sur le discours périgraphique dans la littérature moderne et contemporaine, Gérard Genette consacre un chapitre particulier à la postface. Il y constate d'abord qu'alors qu'il s'attendait «à trouver un corpus de postfaces originales presque aussi copieux que celui de la préface ", il n'en a rien été. Et il considère comme très significatif « la minceur flagrante de ce corpus ». Il convient donc d'expliquer cette pénurie :

La raison essentielle me parait finalement fort claire : placée en fin de livre, et s'adressant à un lecteur non plus potentiel, mais effectif, la postface est certes pour lui de lecture plus logique et plus pertinente. Mais pour l'auteur, et d'un point de vue pragmatique, elle est néanmoins d'une efficacité beaucoup plus faible, puisqu'elle ne peut plus exercer les deux types de fonctions cardinales que nous avons trouvés à la préface : retenir et guider le lecteur en lui expliquant pourquoi et comment il doit lire le texte. Faute de la première action, il n'aura peut-être jamais l'occasion d'arriver jusqu'à une éventuelle postface ; faute de la seconde, il sera peut-être trop tard pour redresser in extremis une mauvaise lecture déjà faite. Par son emplacement et son type de discours, la postface ne peut espérer exercer qu'une fonction curative, ou corrective.

Ceci expliquant, selon Genette, la préférence des écrivains pour la préface, ses difficultés et ses gaucheries mais «dont les vertus sont du moins, et à ce prix, monitoires et préventives » Et celui-ci de conclure : « Pour la postface, il est toujours à la fois trop tôt et trop $\operatorname{tard}^{4} . »$

6 Voyons si les postfaces à Qui se souvient de la mer et à La Nuit sauvage, de nature à infirmer les statistiques de Genette (mais Dib n'a pas droit de cité dans son essai), confirment au moins mon jugement.

\section{Qui se souvient de la mer (1962), une postface pédagogique}

7 Lorsqu'en 1959-60, en pleine guerre d'Algérie, il se lance dans l'écriture de ce roman, Mohammed Dib est à la recherche d'une nouvelle forme: écrivain de conviction et 
d'engagement, qui restera toute sa vie taraudé par une question: celle de la responsabilité de l'intellectuel et de l'artiste, il n'est pas pleinement satisfait de la forme donnée à ses idées dans la trilogie Algérie, qui a pourtant été un succès de librairie mais dont Naget Khadda a bien montré qu'il s'agissait moins de romans au goût dibien que d'une "réponse littéraire à une interpellation idéologique " ${ }^{5}$, comme dans les nouvelles qu'il a jusqu'alors multipliées dans diverses revues, puis recueillies dans Au Café (1955). Cette insatisfaction - il l'a clairement exprimée dans des lettres à plusieurs de ses amis tient au fait que son propos de témoignage, sa volonté, dans les premiers écrits « de marquer une étape dans de la prise de conscience des fellahs algériens ", ainsi qu'il l'énonce à Aragon ${ }^{6}$, ne lui paraît pas à la hauteur de «l'importance du problème ». Le problème en question étant bien évidemment la condition du fellah algérien et la situation dans les campagnes de la colonie $^{7}$; plus globalement ce que l'on va progressivement appeler, dans les cercles politiques, "le problème algérien ». Or, voilà que ce problème vient à s'aggraver à la dimension d'une guerre. Mais comment trouver une forme à la hauteur de celui-ci, si souvent abordé que toutes les relations qui en sont faites, quelles qu'en soit la forme, sont depuis longtemps bourrées de poncifs?

\section{Explication de texte}

8 En 1959 et 60, après la publication d'Un été africain, première tentative pour aborder sous une forme déjà différente la guerre d'Algérie, Dib s'éloigne de sa région natale, non pour la France et un exil, mais pour le Maroc où il va vivre plus d'une année, porté par « de magnifiques projets [...]. Ce n'est pas un film, qui aurait été tiré de mon œuvre, mais trois ${ }^{8}$ !»; période durant laquelle il compose, dans ce hors-lieu qu'est le Maroc $^{9}$ déjà indépendant et dans des conditions assez inconfortables, Qui se souvient de la mer, son cinquième roman révélant une nouvelle approche de la guerre (déjà abordée dans diverses nouvelles et dans Un Eté africain), mais cette fois selon une esthétique tout à fait novatrice où une prose poétique (type d'écriture que Dib a toujours visé), qui sollicite le lecteur et le met à l'épreuve, se substitue brutalement à l'écriture référentielle et transparente qui facilitait la lecture des premiers romans : «Pour dire la guerre, Dib renonce au mimétisme de l'écriture réaliste qui lui semble inefficace à rendre compte d'une expérience des limites et opte pour les visions cauchemardesques qu'autorise le code fantastique ${ }^{10} »$.

Et que peut faire un romancier, jusqu'alors peu satisfait de son art, lorsqu'il sait qu'il aborde dans une forme exigeante un thème aussi rebattu que la guerre et qu'il voit bien (Dib a lu les romanciers américains, les populistes: Poulaille, Guilloux, sans doute les romanciers de la Grande guerre, les réalistes arabophones d'Egypte ${ }^{11}$ ) d'une part, que le réalisme littéraire sera ici de peu de poids? d'autre part, que la vision nouvelle qui s'est imposée à lui a toutes les chances de dérouter ? sinon expliquer sa démarche et justifier à travers l'exemple saisissant du Guernica de Picasso («Pas un élément réaliste dans ce tableau - ni sang, ni cadavres - et cependant il n'y a rien qui exprime autant l'horreur. ») l'abandon nécessaire des formes dans lesquelles le romancier s'est jusqu'alors exprimé ? C'est donc cela qu'il va chercher à démontrer dans sa postface, qui fonctionne comme « une sorte de semi condamnation du réalisme, impuissant à rendre la vraie dimension de l'horreur $»^{12}$. Donc, par voie de conséquence, semi condamnation de ses romans antérieurs... 


\section{Dialogue avec le lecteur}

9 Et puis il y a - me semble-t-il - un autre niveau de lecture de cette postface comme telle (i.e. écrite après), qu'évoque également Genette, mais lui comme une des vertus cardinales de la préface : guider le lecteur. A la suite d'un changement d'esthétique aussi profond, Dib est en droit de se demander si son lecteur, qui a accroché à la lecture des romans antérieurs, va continuer à le suivre. Qui se souvient de la mer paraît fin août 1962, sans sa postface, semble-t-il. Les Lettres françaises d'Aragon et de Jean Ristat en rendent compte les premiers (23 août), puis vient la presse communiste, Le Canard enchaîné (Robert Escarpit). En octobre, une lettre d'Emmanuel Roblès, directeur de la collection dans laquelle le roman est paru, serait de nature à rassurer Dib: son livre se vend correctement et on va pouvoir envisager un retirage. Mais, suspectant « un malentendu, que pour ma part [il] ne saisi[t] pas ", Dib lui répond: "Le succès de Qui se souvient de la mer, dont vous vous réjouissez si amicalement, me surprend quant à moi. Il m'a semblé avoir écrit un livre assez difficile, plus fait pour décourager le lecteur que pour l'attirer. » 13

10 C'est alors qu'il lui propose ces quelques pages de postface, qui se présentent comme un dialogue, une réponse à un courrier de lecteur : «A la question qui m'a été posée - et que chaque lecteur peut légitimement se poser : pourquoi dans ce nouveau roman, le drame algérien m'a poussé à prendre pareil ton et à mettre ses grandes années de malheur dans un cadre terrible et légendaire, je ne sais trop aujourd'hui que répondre... » Ces phrases s'enchaînent ensuite avec l'explication de texte: comparative, puisque Dib fait ici dialoguer sa manière d'écrire avec le Guernica de Picasso.

On a donc affaire ici à une "postface ultérieure ", ou "postface tardive", selon la dénomination de Genette - une postface écrite en réaction à (ou en prévision de...) des difficultés, des réticences de réception et dont « la grande affaire est de répondre aux critiques formulées par la réception.

\section{La Nuit sauvage (1995)}

11 La postface de La Nuit sauvage (1995) est une « postface originale » selon la terminologie de Genette, c'est à dire que, contrairement à celle de Qui se souvient de la mer, elle accompagne le texte dès sa première édition. Elle obéit de ce fait à une stratégie toute différente : réflexion sur la littérature et certains de ses genres, elle est aussi guidée par un principe de cohérence.

\section{Le Principe de cohérence}

12 Le principe de cohérence, défini par Genette, consiste à essayer de montrer par une préou postface la cohérence d'un ensemble ou d'un texte qui, à première lecture, pourrait paraitre disparate.

Cohérence d'abord du recueil : Dib a écrit un grand nombre de nouvelles; il aimait cette forme brève qui combine la fulgurance poétique et la durée du roman; il en a fait le laboratoire de certains de ses romans et des critiques comme Naget Khadda ou Charles Bonn ont su démontrer la continuité existant entre certaines de ces nouvelles et les romans. Le plus grand nombre d'entre elles devra être retrouvé dans des revues ou peutêtre à l'état de manuscrits dans ces archives déposés à la BnF. Certaines, retravaillées, ont 
intégré tel ou tel roman. Il faudra aussi examiner les modalités de ce processus de réintégration. Enfin, il y a trois recueils soigneusement réunis et organisés par l'auteur : Au café (1955), Le Talisman (1966) et La Nuit sauvage (1995).

Dans cette postface, Dib s'applique tout d'abord à montrer non seulement la cohérence interne de ce dernier recueil, mais surtout le rapport que ces nouvelles entretiennent avec ses romans antérieurs, leur ensemble pouvant être tenu « pour un roman qui, fait de rejets issus du tronc commun et lié par les mêmes affinités, devrait aller à son tour grossir le maître-roman?» Revenant là à l'une des vertus cardinales attribuées par Genette au discours pré/postfaciel, celle de guider le lecteur, l'auteur nous fournit une clef de lecture, "un point de vue à partir duquel, après tout pourraient se lire les nouvelles de ce recueil, encore que chacune se suffise à elle-même et justifie une lecture à part entière. »

\section{Une réflexion sur la littérature}

Cette notion de cohérence conduit progressivement l'auteur à une réflexion sur «la continuité de [tout] récit [qui] ne représente à l'origine qu'un conglomérat, une galaxie de micro-récits » - véritable alchimie dit-il, apanage du lecteur plus que de l'auteur, mais dont nous ressentons bien davantage la continuité que nous ne la comprenons :

On ne voit pas comment fonctionne la continuité tout simplement parce que nous sommes ses prisonniers; je crois. Le rêveur sous l'emprise de son rêve ne s'étonne pas des ruptures, des failles, des solutions de continuité qui jalonnent l'aventure onirique; il n'est sensible qu'à la cohérence sous-jacente des péripéties, la seule à faire sens à ses yeux. Ecrivant ou lisant, nous vivons aussi un rêve.

Elle conduit aussi à une question qui a toujours travaillé Dib: la responsabilité de l'écrivain: «A quelle interrogation plus grave que celle de sa responsabilité, un écrivain pourrait-il être confronté?». Nos sociétés modernes disjoignent écriture et responsabilité. Sans incliner pour les intellectuels donneurs de leçon, Dib pense «qu'on ne peut pas et qu'on ne doit pas. » Et que si l'écrivain se sait désormais incapable «d'apporter des réponses » aux grands maux de l'humanité, cela ne doit pas le dispenser de continuer à interroger le monde, à « apporter des questions ».

On mesure tout à la fois la distance et l'intérêt entre ces deux postfaces de Dib. La première, écrite à 40 ans, prospective et interrogative : comment dire encore - puisqu'il le faut - après les tranchées, la Shoah, la guerre civile en Espagne, l'un des maux les plus cruels du XXe siècle? La seconde, que l'écrivain clôt à 75 ans, conclut l'un des derniers ouvrages de cette œuvre immense en forme de bilan, de réflexion sur une œuvre et sur la littérature en général.

\section{Aragon : préface à Ombre gardienne}

Reste une préface allographe : il s'agit de celle qu'Aragon a offerte à Dib pour son premier recueil poétique, Ombre gardienne (Gallimard, 1961). Bien que nombre de préfaces soient comme ici la conséquence d'une longue amitié, entretenue de lectures réciproques et d'estime, je ne voudrais pas non plus m'étaler trop longuement sur la relation entre Dib et Aragon, basée sur une estime réciproque et de semblables engagements : une quinzaine de lettres retrouvées entre 1954 et 1962, et au moins une rencontre en 1959, donc peu de temps avant la publication d'Ombre gardienne. Mais pour comprendre la préface d'Aragon 
à ce recueil poétique, on doit extraire quelques éléments de cette correspondance. A l'auteur de La Semaine sainte, désireux de rendre compte de ses deux premiers romans dans Les Lettres françaises, Dib répond en juin 1954 :

Il me semble pour le moins prématuré d'accorder beaucoup de l'importance à ce que j'écris. Écrivant, j'y mets tout mon cœur, et c'est pourquoi sans doute j'éprouve tant de difficulté à écrire. Toutefois, j'ai beaucoup lu et je vous assure que je sais très bien ce que valent mes deux livres : peu de chose, même dans l'honnête moyenne et banale moyenne. On peut déceler dans les auto-jugements fréquents de Dib dans ses lettres à Aragon, comme dans celles à d'autres de ses aînés, Audisio ou Roblès, ce manque de confiance d'un jeune écrivain en son art romanesque que j'ai relevé au début de mon article, mais on doit y voir aussi deux autres choses :

- D'abord une préférence marquée pour l'écriture poétique par laquelle il a débuté ("Vega », 1946) et à travers laquelle il admet davantage d'être jugé. Surtout par Aragon, en qui il voit un maitre inégalable du vers et du verbe.

- Ensuite une certaine coquetterie, et qui n'est pas propre à Dib: Sartre dont il se réclamait beaucoup offrit le choix à Albert Memmi de rendre compte de son Portrait $d u$ colonisé dans Les Temps Modernes ou de le préfacer. Lorsqu'il lui posa la question «Préférez-vous ceci ou cela? ?, le jeune écrivain lui répondit : «Les deux! » Et pourquoi pas?

17 Il en va de même pour Aragon et Dib : des années durant, l'aîné entend écrire sur l'œuvre romanesque ou les nouvelles de l'écrivain algérien. Mais celui-ci minaude, objecte que sa prose ne mérite pas un tel honneur, qu'il ne croit pas «mériter tant » et qu'il a " peur qu'il n'y ait disproportion entre ce livre et l'intérêt » que lui porte Aragon, que l'écho qu'une préface de lui serait ressenti comme excessif et injuste : «Il aurait suffi que vous, le plus grand écrivain français actuel, parliez de quelqu'un comme moi, et quels que soient les termes dans lesquels vous le fassiez, pour que la différence éclate, paraisse exagérée, pour que mon livre échappe à ses justes proportions qui sont, à vrai dire, modestes. $»^{14}$

Pourtant, après un ou deux articles d'Aragon sur les premiers romans de Dib, qui lui permettent de saisir qu'en « Un roman commence » (Les Lettres françaises, 8 juillet 1954), des articles dont celui-ci ne retient que les critiques (« Vous avez exprimé des réserves. $C^{\prime}$ est de cela aussi que je voudrais savoir vous remercier. Vous renforcez ainsi ce que je pensais de mes propres écrits $»^{15}$ ), c'est au contraire Dib lui-même qui sollicite Aragon pour cette préface d'Ombre gardienne, son premier recueil poétique. Comme si la poésie constituait l'espace de son irréductible singularité, qu'entre poètes il pourrait enfin lui confier :

J'aimerais pouvoir vous donner ce manuscrit en mains propres. Ne m'en veuillez pas d'insister de la sorte. C'est une chose très importante pour moi ; il y dans ces vers quelque chose que vous seul pourriez comprendre. De plus, nous écrivains algériens, nous sommes traités un peu en parents pauvres de la langue française. Tant qu'il s'agit de la prose, on peut avoir raison contre nous. Mais voici des vers, de la poësie, ce qu'il y a donc de plus insaisissable du génie d'une langue. Et là-dessus, vous, un maître de la langue française, avez votre mot à dire ${ }^{16}$.

Et Aragon de se mettre au diapason dans sa préface, découvrant dans l'usage du français chez ces voleurs de feu :

un aspect essentiel de ce drame algérien, sur quoi tant de gens de chez nous se prononcent avec une certaine légèreté, qu'à l'heure de l'expression la plus haute, ceux-là même qui sont la fidélité à son 
peuple aient pour langage, pour atteindre à ce qui échappe à l'analyse, pour exprimer l'incernable, ce français, ce clavecin bien tempéré, cet instrument des bords de Loire, ce parler qui est aussi celui des soldats dans la nuit, ce vocabulaire du ratissage, le commentaire de la torture et de la faim.

Mais pour le lecteur français, «Comment [...s]'y prendre? Puis-je de mes yeux français saisir la naissance de la poésie algérienne ? Le roman, toujours, le conte, la nouvelle, c'est comme une invitation au voyage : j'entre avec l'auteur dans son Algérie inconnue. Mais le poème ${ }^{17}$ ? »

\section{En guise de conclusion}

Alors... que nous disent ces deux postfaces de Dib à ses propres œuvres, un roman et un recueil de nouvelles, cette préface d'Aragon à Ombre gardienne? Et ces correspondances à l'appui ? Comment les considérer dans une édition génétique qui doit tenir compte du dynamisme de toute œuvre en train de se faire (celle de Dib supposant, qui plus est, une configuration bien particulière) et en même temps son ouverture au contexte?

\section{NOTES}

1. Au sens qu'Antoine Compagnon donne à cette expression dans La Seconde main ou le travail de la citation (éd. Seuil, Paris, 1979), p. 328 : « la périgraphie est une zone intermédiaire entre le horstexte et le texte. Il faut passer par elle pour accéder au texte. Elle échappe un tant soit peu à l'immanence du texte, non qu'elle lui soit transcendante, mais elle le situe, le met en place dans l'intertexte, elle témoigne du contrôle que l'auteur exerce sur lui. C'est une scénographie qui met le texte en perspective, et l'auteur en est le centre. »

2. Ibidem, p. 344-45.

3. Éditions du Seuil, 1987. Coll. "Points Essais", p. 240-242.

4. Ibidem.

5. Titre de l'article qu'elle donne au colloque de Nice : Question coloniale et écriture, recueilli dans Les Carnets de l'exotisme, $\mathrm{n}^{\circ}$ 14, janvier-juin 1995, p. 29-40. Aragon qui suit Dib depuis ses débuts parle alors d' « Un roman qui commence », Les Lettres françaises, 8 juillet 1954.

6. Lettre du 8 mars 1954, quelques jours avant la sortie de L'Incendie, qu'il définit comme « un roman qui n'en est pas un » et dont il dit : «Il m'a plutôt l'air d'un échec ».

7. Notamment la faim; tout début des années 50, Dib s'est déplacé à Alger pour faire des conférences à ce sujet.

8. La période assez mystérieuse dont personne ne parle mais sur laquelle il faudra se pencher s'il y a quelque chance de retrouver toutes les archives manquantes dont Qui se souvient de la mer est le dernier maillon : «j'ai tout laissé à Tlemcen », répond-il à Roblès qui le presse d'écrire sur Camus à la mort de celui-ci.

9. À Roblès, Dib rapporte qu'il y rencontre Sefrioui et Mammeri, lui-même en pleine rédaction de L'Opium et le bâton, vision de la guerre d'Algérie qui se situe à l'opposé de celle que présente Dib dans Qui se souvient de la mer.

10. Khadda, Naget, Mohammed Dib, cette intempestive voix recluse, Edisud, Aix-en-Provence, 2003, p. 130. 
11. ... mais aucun livre de science-fiction, comme il le signale dans sa postface !

12. Charles Bonn : «Les Pouvoirs du langage », Mohammed Dib. Itinéraires et contacts des cultures, $\mathrm{n}$ - 21-22, 1995, p. 152.

13. Lettre à Emmanuel Roblès, Mougins, le 5 novembre 1962.

14. Lettre de Dib à Aragon, 3.VI.1954, op. cit.

15. Lettre de Dib à Aragon,

16. Lettre de Dib à Aragon, Paris le 13 juin 1960.

17. Préface d'Aragon à Ombre gardienne, réédition Sindbad, 1984, p. 12.

\section{RÉSUMÉS}

L'étude du discours péritextuel peut et devrait constituer un passage obligé pour chaque généticien. Qu'il s'agisse d'une préface ou d'une postface, qu'elle soit autographe ou allographe, elle peut en dire long du processus d'écriture et des relations entre les écrivains. Les exemples à l'appui (deux postfaces de M. Dib et une préface de L. Aragon) en font preuve.

Studying the peritextual discourse could and should be a compulsory stage for all researcher practising genetic criticism. Il does not matter whether it is an introduction or an afterword, whether it has been written by the author himself or by someone else; it can say a lot about the writing process and about the relations between writers. The examples quoted (two afterwords by M. Dib and one introduction by L. Aragon) are clear support to this statement.

\section{AUTEUR}

\section{GUY DUGAS}

Professeur émérite université Montpellier III - ITEM 